\title{
Measurement of skin viscoelasticity: A non-invasive approach in allergic contact dermatitis
}

\author{
MARIA-MAGDALENA CONSTANTIN ${ }^{1,2^{*}}$, STEFANA BUCUR $^{1^{*}}$, ELENA-DANIELA SERBAN $^{1}$, \\ RODICA OLTEANU ${ }^{1}$, OVIDIU GABRIEL BRATU ${ }^{2,3}$ and TRAIAN CONSTANTIN ${ }^{2 *}$ \\ ${ }^{1}$ The Second Department of Dermatology, Colentina Clinical Hospital, 020125 Bucharest; \\ ${ }^{2} 3$ rd Department, 'Carol Davila' University of Medicine and Pharmacy, 050474 Bucharest; \\ ${ }^{3}$ Urology Department, Emergency University Central Military Hospital, 010825 Bucharest, Romania
}

Received June 15, 2020; Accepted July 15, 2020

DOI: $10.3892 / \mathrm{etm} .2020 .9314$

\begin{abstract}
Non-invasive bioengineering technologies are constantly being developed, as they can provide useful insights and contribute to the improvement of medical care and scientific education. The purpose of this study was to assess skin viscoelasticity using the suction chamber method in patients with allergic contact dermatitis vs. healthy subjects, before and after applying a moisturizer safety testing cream. This was a prospective controlled study over a 3-year period (March 2016-March 2019), with 81 subjects being divided in two balanced groups: Patients with allergic contact dermatitis and healthy subjects, respectively. The skin viscoelasticity was determined for all subjects with Cutometer ${ }^{\circledR}$, using the suction method, by performing a dynamic assessment of parameters before and after applying a moisturizing cream. The results indicate a decrease in the elasticity parameters in both groups, indicating an improvement of the elastic properties under the treatment. Skin capacity to return to its previous form after the deformation, i.e., pure elasticity and biological elasticity, showed overall elevated values in the group with contact dermatitis, demonstrating the efficacy of the emollient cream after applying it for 28 days (increase by 11.7 and $4.9 \%$ respectively, compared with baseline, when patients had dry, untreated skin). However, in healthy subjects, these parameters do not achieve important values, but they remain rather stable over time with a very slight improvement $(6.6 \%$ after 28 days). The Cutometer is an easy to use, efficient and widely used instrument for measurements in studies that perform a
\end{abstract}

Correspondence to: Dr Stefana Bucur, The Second Department of Dermatology, Colentina Clinical Hospital, 19-21 Stefan cel Mare Blvd., 020125 Bucharest, Romania

E-mail: stefanabucur11@gmail.com

\section{${ }^{*}$ Contributed equally}

Key words: cutometer, skin barrier, viscoelasticity, safety testing, formulation, allergic contact dermatitis quantitative assessment of the effectiveness of different formulations intended for application on the skin.

\section{Introduction}

The mechanical properties of the human skin have been studied in the past proving that elasticity is a dynamic function (1). More precisely, it has the properties of both elastic solids and viscous liquids (2).

Non-invasive bioengineering technologies are constantly being developed with the potential of offering new mechanistic insights. The Cutometer ${ }^{\circledR}$ (Courage+Khazaka Electronic $\mathrm{GmbH}$ ) has been introduced as a device that can measure the viscoelastic properties of the skin in vivo. It provides information on physiological and pathological changes of human skin as well as the efficacy of topical treatment.

The present study assessed the viscoelasticity of the skin in a group of patients with chronic allergic contact dermatitis vs. a control group of healthy individuals. Assessment was performed after applying a moisturizer to both groups, demonstrating the effectiveness of this moisturizer for both healthy and unhealthy skin.

\section{Patients and methods}

This was a prospective controlled study over a period of 3 years (March 2016-March 2019), on a group of patients diagnosed with allergic contact dermatitis, the chronic type, with recurrent episodes (ACDG) and on healthy subjects (HSG). All the subjects signed an informed consent form. The inclusion criteria were: Age between 18 and 70 years with no previous treatment; allergic condition confirmed by the previous positive epicutaneous tests for specific allergens (patch-testing) for ACDG specifically (3). The exclusion criteria were: Known history of allergy to ingredients included in the testing cream; other diagnosed skin diseases $(4,5)$; malignant skin tumors in the last 5 years $(6,7)$; recent vaccinations, 2 weeks before starting the study or the intention of vaccination during the study; recent dermato-cosmetic or esthetic treatments, 2 months before the onset of the study (dermabrasion, peeling, laser therapy, IPL, botulinum toxin injections or fillers); recent and intense exposure (1 month before the study) to ultraviolet 
Table I. Formula of the moisturizing cream.

\begin{tabular}{ll}
\hline Composition & \multicolumn{1}{c}{ Role } \\
\hline Aqua & Vehicle \\
Paraffin & Emollient \\
Glyceryl (mono)stearate & Emulsifier \\
Peg 100 stearate & Emulsifier \\
Glycerin & Humectant; NMF \\
Isopropyl myristate & Emollient \\
Petrolatum & Emollient \\
Cetyl alcohol & Emollient \\
Palmitic stearic acid & Emulsifier \\
Paraffinum liquidum & Emollient \\
Cera alba & Emollient \\
Sodium benzoate & Preservative \\
Parfum & Perfume \\
Sodium polyacrylate & Stabilizer \\
Prunus Amygdalus Dulcis Oil & Active principle \\
Xantan gum & Curing agent \\
Urea & Humectant \\
Sodium lactate & Humectant \\
Panthenol & Active principle \\
Tocopheryl acetate & Active principle \\
Sodium hydroxide & pH adjuster \\
Citric acid & pH adjuster \\
Bisabolol & Active principle \\
& \\
&
\end{tabular}

$\mathrm{NMF}$, natural moisturizing factor.

Table II. Main parameters of viscoelasticity.

Uf-final deformation: Distensibility

Ue-immediate deformation: Extensibility

Uv-viscoelastic contribution: Plasticity

Ur-immediate retraction

Ur/Ue-pure elasticity

Ur/Uf-biologic elasticity

radiation (sun/tanning salon); pregnancy of intention of pregnancy; inability to follow protocol for any reason.

A total of 81 individuals were divided in two balanced groups, the HSG with 41 individuals and the ACDG with 40 individuals, respectively.

The evaluation of cutaneous elasticity was performed on the anterior side of the middle third of the left forearm. Measurements were done before and after applying a moisturizing cream twice a day for 28 days at: Baseline-T0, one hour after application-T1h and 28 days after-T28d. After using the moisturizer, the same parameter was evaluated again dynamically, at certain time frames. The moisturizer used in the study was an oil-in-water cream (compounds listed in Table I).

The measurements were carried out using the suction method with the help of Cutometer ${ }^{\circledR}$ MPA 580, made by Courage + Khazaka Electronic GmbH in Germany, under

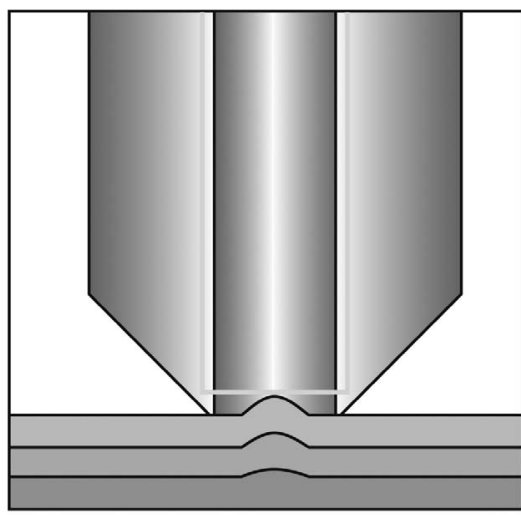

Figure 1. Elasticity measurement probe.

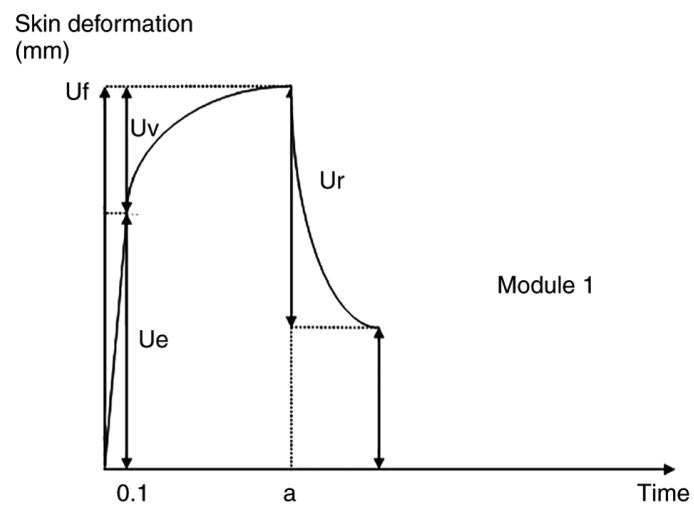

Figure 2. Cutaneous biomechanical properties represented by a curve of deformation according to time.

standard conditions of temperature and humidity $\left(\mathrm{T}=20-22^{\circ} \mathrm{C}\right.$, humidity $40-60 \%$ ) and after a rest period of $15-20 \mathrm{~min}$ for each subject tested (8).

Cutaneous elasticity, defined as the skin property of regaining the shape after stretching, can be measured through many non-invasive methods. The method used in the present study is based on the principle of suction and it is realized with a probe (Cutometer MPA 580). This probe measures cutaneous changes and can evaluate the efficacy of some topical products. The exploration probe makes a vacuum through a suction obtained at a constant negative pressure (450 mbar) (Fig. 1). The skin is absorbed in the probe's aperture $(2 \mathrm{~mm})$ for an established period of time, ascends to a maximum level, followed by a relaxation period and the skin returns to the initial level. The level difference, expressed in $\mathrm{mm}$, between the two points is processed and expressed as a percentage. The variation ranges are: Less than $0.5 \mathrm{~mm}$, low elasticity; between 0.5 and $1.5 \mathrm{~mm}$, normal elasticity; over $1.5 \mathrm{~mm}$ increased elasticity (Courage + Khazaka Electronic GmbH, 2020. www.courage-khazaka. de/index.php/en/products/scientific/140-cutometer). At every measurement, the skin biomechanical properties are represented by a curve of deformation according to time (Fig. 2).

The principle of the suction method is measuring skin elevation produced by the suction force exerted over a defined area of the skin (9). Measuring suction time or suction force serves to calculate various parameters of skin mechanical properties (Table II) (10). 


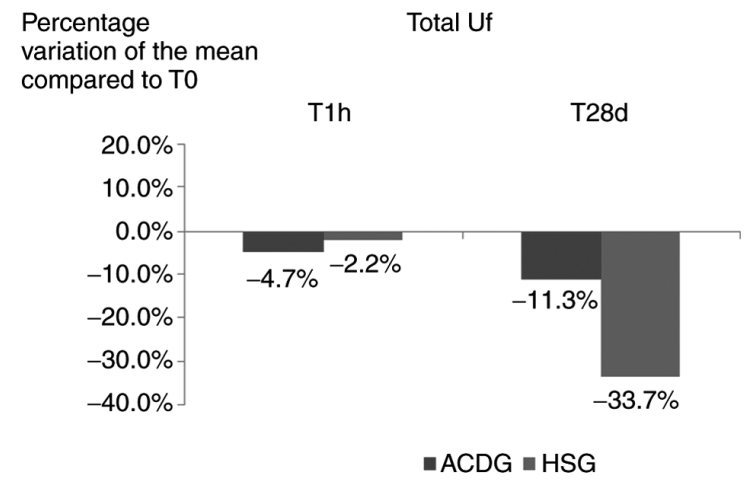

Figure 3. Final deformation (Uf).

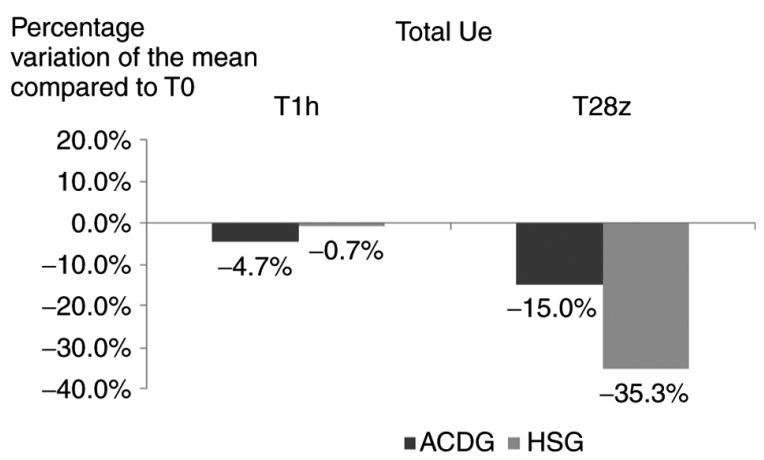

Figure 4. Immediate deformation or extensibility (Ue).

Skin elasticity is reflected by the $\mathrm{U}$ and $\mathrm{R}$ parameters, defined as: Ur (immediate retraction), Ue (immediate deformation), Uf (final deformation), Ur/Uf (biological elasticity), $\mathrm{Ur} / \mathrm{Ue}$ (net elasticity without viscous deformation) and Ua/Uf (gross elasticity including viscous deformation). These parameters are related to the function of elastic fibers and reflect their changes in physiological or pathological skin conditions, while the viscoelasticity of the skin is related to the displacement of interstitial fluid through the fiber network in the dermis and is reflected by the parameters Uv (delayed deformation), Uv/Ue (viscoelastic ratio, i.e., the ratio of viscoelastic to elastic distension) and Ua (called also R8, meaning the final retraction after removal of the vacuum) (11).

Variations of $4 \mathrm{U}$ and $\mathrm{R}$ parameters were analyzed: Uf, Ue, $\mathrm{Ur} / \mathrm{Ue}$ and Ur/Uf in both groups. The measurements are illustrated by curves that translate the ability of the skin to return to its initial shape (elasticity) and its mechanical strength to traction (firmness). Mechanical properties of the skin, and therefore its elasticity, can be influenced by several biological or environmental factors including: Race, age, sex of the volunteers, anatomical area, water balance of the organism, hormones, comorbidities, circadian rhythm, photo exposure, season, physical stress and topical treatments (12).

Results are presented as the mean \pm SD or median and 25 th to 75 th, as appropriate for normality of data. We used the Mann-Whitney U test or unpaired Student's t-test for continuous and independent data and the paired Student's t-test for paired data. The Pearson's correlation coefficient was used to assess correlation between continuous variable. $\mathrm{P}<0.05$ was considered to indicate a statistically significant
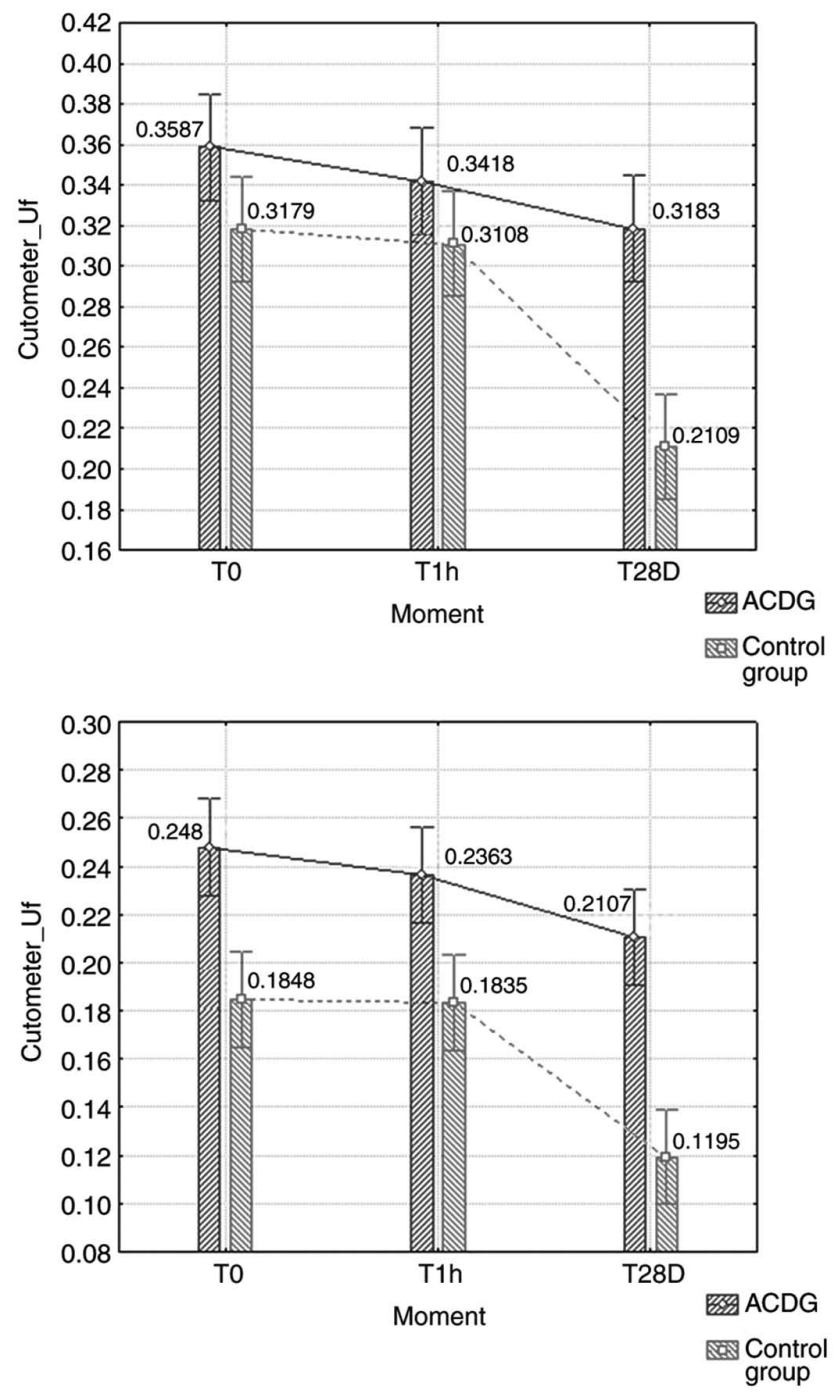

Figure 5. Uf and Ue variation, Mann-Whitney, Student T, $\mathrm{P}=0.0120$ for Uf, $\mathrm{P}<0.001$ for Ue.

difference. Statistical tests were performed using SPSS software v15.

Day 7 evaluation is not reported here, as no relevant changes from the previous evaluation were observed.

\section{Results}

Final deformation (Uf) in both groups decreased from baseline by $4.7 \%$ for ACDG and $2.2 \%$ for HSG one hour after emollient application. At 28 days after treatment, this decrease was higher compared with the previous times, but it was also reversed between the two groups (11.3\% ACDG vs. 33.7\% HSG) (Fig. 3).

Immediate deformation or extensibility (Ue) in both groups decreased less in one hour after emollient application (4.7\% ACDG vs. $0.7 \%$ HSG) and more after 28 days of treatment (15.0\% ACDG vs. 35.3\% HSG) (Fig. 4).

Both parameters of the skin deformation, Uf and Ue, showed a parallel decrease in both groups (Fig. 5). However, this decrease was more important in the HSG $(\mathrm{P}=0.012$ for Uf and $\mathrm{P}<0.001$ for $\mathrm{Ue}$ ).

The Ur/Ue ratio (net elasticity without viscous deformation, i.e., pure elasticity) increased in the ACDG group at one hour 


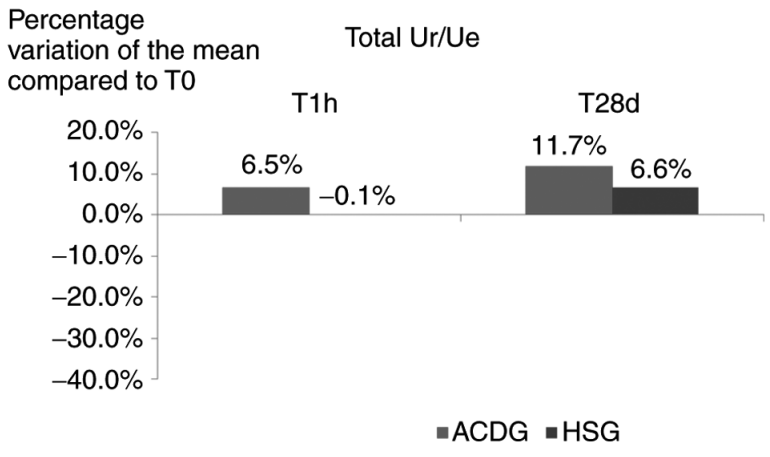

Figure 6. Variation of pure elasticity.

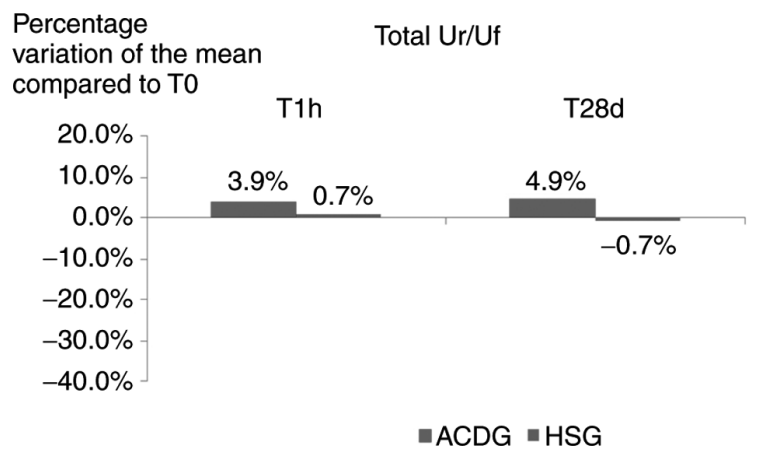

Figure 7. Variation of biological elasticity.

(6.5\%) and 28 days $(11.7 \%)$ post-application of moisturizing with the baseline T0 (Fig. 6). In the HSG, there were no differences in measurements between baseline and $\mathrm{T} 1 \mathrm{~h}$, however differences occurred after 28 days (6.6\%).

The Ur/Uf (the biological elasticity) had similar values in the HSG each time at the 3 time points considered. For the ACDG there were very slight increases, approximately equally when comparing the values at $\mathrm{T} 1 \mathrm{~h}$ and $\mathrm{T} 28 \mathrm{~d}$, with baseline $\mathrm{T} 0$ (Fig. 7).

To sum up, in the ACDG the Ur/Ue (net elasticity without viscous deformation) increased progressively from T0 to T28d, as well as Ur/Uf (biological elasticity). In the HSG, $\mathrm{Ur} / \mathrm{Ue}$ stagnated at $\mathrm{T} 0$ and $\mathrm{T} 1 \mathrm{~h}$, but it had a slight increase at T28d. Ur/Uf showed almost a plateau; there was a mild change in the $\mathrm{Ur} / \mathrm{Ue}$ variation $(\mathrm{P}=0.5773$ for $\mathrm{Ur} / \mathrm{Ue}$ and $\mathrm{P}=0.0765$ for Ur/Uf) (Fig. 8).

\section{Discussion}

Skin mechanical functions are the expression of the biomechanical nature of its components and structure. Elasticity depends on the thickness of the skin and on the area where it is measured. Previous studies showed that there are important differences in mechanical properties of the skin relating to body site dominant/non-dominant sides or aging $(12,13)$. Moreover, sex hormones seem to have a significant influence in the mechanical properties of the skin and therefore its elasticity (14).

In this study, skin elasticity was assessed using the $\mathrm{U}$ and $\mathrm{R}$ parameters. There are studies implying that the Q-parameters are more accurate than the $\mathrm{U}$ and $\mathrm{R}$ parameters described
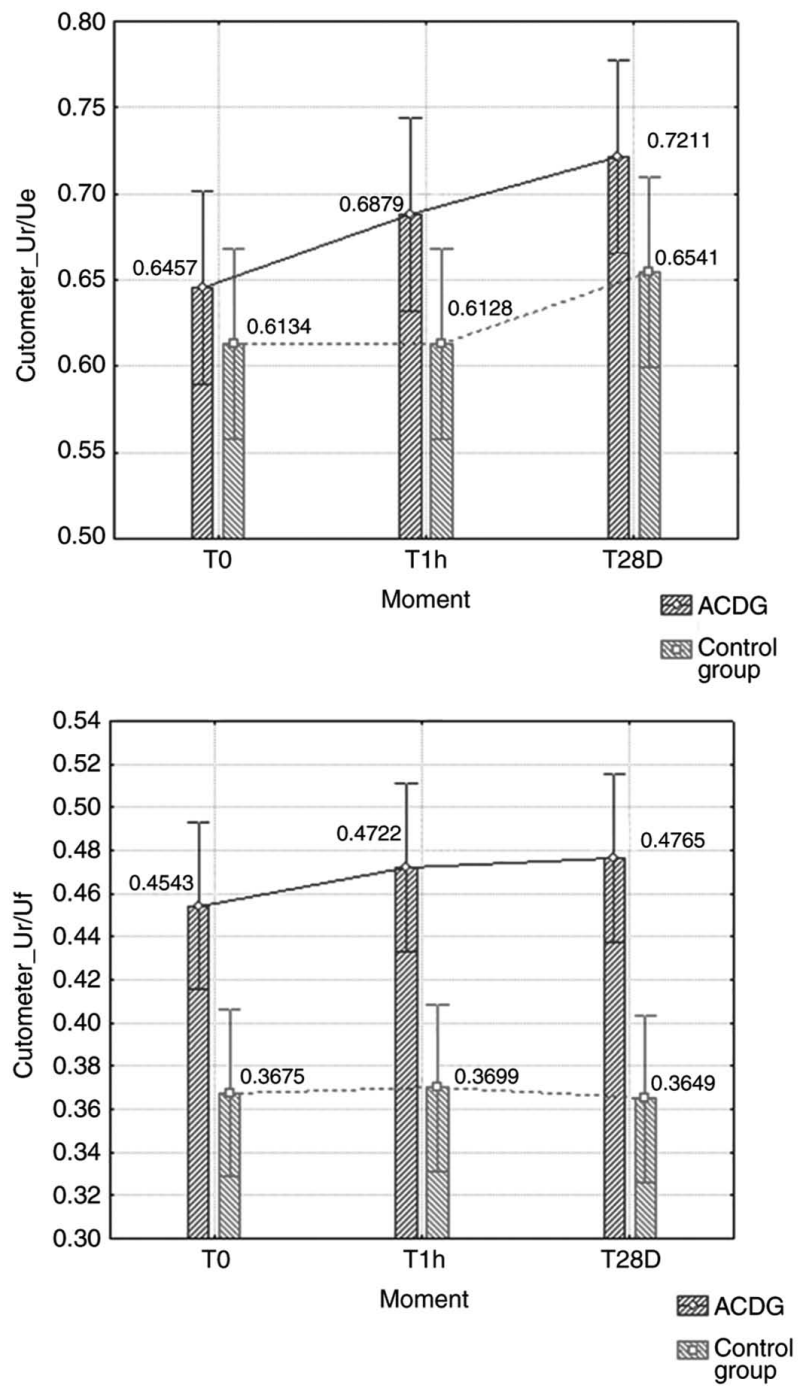

Figure 8. Global analyses, Mann-Whitney, Student T, P=0.5773 for Ur/Ue, $\mathrm{P}=0.0765$ for $\mathrm{Ur} / \mathrm{Uf}$.

above and should be used to improve accuracy of measurement of the human skin elasticity (15).

The main findings showed a decrease in the Uf and Ue parameters in both groups, demonstrating that skin elastic properties improved under the treatment. More precisely, after 28 days, in the ACDG, the final deformation (Uf) decreased by $11.3 \%$ and the final retraction (Ua) by $15 \%$, respectively.

The ratios that define the skin's ability to return to its previous form after deformation, pure elasticity (Ur/Ue) and biological elasticity (Ur/Uf), increased in the ACDG, proving the efficacy of the emollient cream over this mechanical parameter after its application for 28 days (Ur/Ue increased by $11.7 \%$ and Ur/Uf by $4.9 \%$ when comparing to baseline in the group of patients with dry, untreated skin). In the HSG, these parameters did not show important values, but remained rather stable over time, with a slight improvement (6.6\% for Ur/Ue at 28 days).

The data obtained indicate an improvement in the firmness of the skin in both groups as a result of the moisturizing effect that the applied emollient cream had. This is in accordance with the previous studies regarding epidermal hydration (16). 
In regard to the cutaneous pathological modification, Cutometer ${ }^{\circledR}$ has proven its use in assessing dermal edema in erysipelas and wound healing process $(17,18)$. Improvement of stretch marks using carboxytherapy was demonstrated with Cutometer (19).

A real help for practitioners and researchers can be the investigation of micromorphological aspects of cutaneous inflammation using in vivo reflectance confocal microscopy (RCM) (20). RCM, Cutometer could be a potential diagnostic tool for cutaneous inflammatory disorders (21). Furthermore, early assessment of inflammation caused by checkpoint inhibitor therapy can predict the type of response to immunotherapy (22).

In conclusion, measurement of the degree of skin viscoelasticity by using the suction method is effective and reproducible and recommends the Cutometer ${ }^{\circledR}$ device as effective and reproducible, non-invasive, in vivo, easy and inexpensive work tool. Cutometer is efficient and widely used in clinical trials for measurements in healthy or pathological conditions, in order to obtain a quantitative assessment of the effectiveness of different formulations intended for application on the skin, under well-controlled and standardized parameters. To support this, a study analyzed skin hydration, elasticity and skin erythema and showed that cosmetic products containing stratum corneum lipids could play a part in the restoration of disturbed skin barrier function (23).

Measuring the parameters analyzed in this investigation may open up new perspectives in the research of topical medication for several diseases, by helping to identify ideal ingredients for desired effects. Bioengineering technologies offer today valuable benefits in research and therapeutic fields; information obtained noninvasively in vivo, rapid results, high objectivity, high reproducibility and the possibility to choose any skin area are just a few advantages of bioengineering technologies.

\section{Acknowledgements}

The authors would like to thank Mrs. Alina Nanu for her great assistance and enthusiastic support in the completion of this study.

\section{Funding}

Not applicable.

\section{Availability of data and materials}

The datasets used and/or analyzed during the present study are available from the corresponding author on reasonable request.

\section{Authors' contributions}

MMC contributed in all the stages of the study, designed the study and revised the manuscript for important intellectual content. EDS and SB contributed to the conception of the study, the revision of the manuscript language and the drafting of the manuscript. RO acquired the data by screening the papers identified on PubMed and drafted the manuscript. TC and OGB analyzed and interpreted the data. All authors read and approved the final version of the manuscript.

\section{Ethics approval and consent to participate}

Patients provided informed written consent prior to their participation to the study.

\section{Patient consent for publication}

Not applicable.

\section{Competing interests}

The authors declare that they have no competing interests and they have no financial relationships to disclose.

\section{References}

1. Piérard GE: A critical approach to in vivo mechanical testing of the skin. In: Lévèque JL (ed) Cutaneous investigation in health and disease, noninvasive methods and instrumentation. Informa healthcare, New York, 1989.

2. Larrabee WF Jr: A finite element model of skin deformation. I. Biomechanics of skin and soft tissue: A review. Laryngoscope 96: 399-405, 1986

3. Constantin MM: Value and impact of patch testing in patients with allergic contact dermatitis. Rev Română de Medicină de Lab 20: 287-292, 2012.

4. Olteanu R,Constantin MM,Zota A, Dorobantu DM,Constantin T, Serban ED, Bălănescu P, Mihele D and Gheucă Solovăstru L: Original clinical experience and approach to treatment study with interleukine 12/23 inhibitor in moderate-to-severe psoriasis patients. Farmacia 64: 6, 2016

5. Olteanu R, Zota A and Constantin M: Biosimilars: An update on clinical trials (Review of Published and Ongoing Studies). Acta Dermatovenerol Croat 25: 57-66, 2017.

6. Solovăstru LG, Vâţă D, Stătescu L, Constantin MM and Andrese E: Skin cancer between myth and reality, yet ethically constrained. Rev Română de Bioetică 12: 47-52, 2014.

7. Iorga RA, Bratu OG, Marcu RD, Constantin T, Mischianu DLD, Socea B, Gaman MA and Diaconu CC: Venous thromboembolism in cancer patients: Still looking for answers (Review). Exp Ther Med 18: 5026-5032, 2019.

8. Constantin MM, Poenaru E, Poenaru C and Constantin T: Skin hydration assessment through modern non-invasive bioengineering technologies. Maedica (Buchar) 9: 33-38, 2014.

9. Serup J: Mechanical properties of human skin: Elasticity parameters and their relevance. In: Elsner P, Berardesca E, Wilhelm KP and Maibach HI (eds). Bioengineering and the skin. Skin biomechanics. CRC Press, Boca Raton, pp41-47, 2002.

10. Agache PG, Monneur C, Leveque JL and De Rigal J: Mechanical properties and Young's modulus of human skin in vivo. Arch Dematol Res 269: 221-232, 1980.

11. Dobrev HP: A study of human skin mechanical properties by mean of cutometer. Folia Med (Plovdiv) 44: 5-10, 2002.

12. Ryu JS, Joo YH, Kim SO, Park KC and Youn SW: Influence of age and regional differences on skin elasticity as measured by the cutometer. Skin Res Technol 14: 354-358, 2008.

13. Smalls LK, Randall Wickett R and Visscher MO: Effect of dermal thickness, tissue composition, and body site on skin biomechanical properties. Skin Res Technol 12: 43-49, 2006.

14. Beiu C, Popescu MN, Dumitrascu MC, Petca A, Mehedințu C, Farcasanu PD, Petca RC, Chibelean C and Sandru F: The evaluation of Estrogen Receptor $\beta$ as a potential prognostic factor in melanoma. Rev Chim (Bucharest) 70: 4212-4216, 2019.

15. Qu D and Seehra GP: Improving the accuracy of skin elasticity measurement by using Q-parameters in cutometer. J Cosmet Sci 67: 37-44, 2016.

16. Dobrev H: Use of cutometer to assess epidermal hydration. Skin Res Technol 6: 239-244, 2000.

17. Dobrev H: Use of cutometer to assess dermal oedema in erysipelas of the lower legs. Skin Res Technol 4: 155-159, 1998. 
18. Held M, Rothenberger J, Tolzmann D, Petersen W, Schaller HE and Rahmanian-Schwarz A: Alteration of biomechanical properties of skin during the course of healing of partial-thickness wounds. Wounds 27: 123-128, 2015.

19. Podgórna K, Kołodziejczak A and Rotsztejn H: Cutometric assessment of elasticity of skin with striae distensae following carboxytherapy. J Cosmet Dermatol 17: 1170-1174, 2018.

20. Ilie MA, Caruntu C, Lixandru D, Tampa M, Georgescu SR, Constantin MM, Constantin C, Neagu M,Zurac SA and Boda D: In vivo confocal laser scanning microscopy imaging of skin inflammation: Clinical applications and research directions. Exp Ther Med 17: 1004-1011, 2019.
21. Căruntu C and Boda D: Evaluation through in vivo reflectance confocal microscopy of the cutaneous neurogenic inflammatory reaction induced by capsaicin in human subjects. J Biomed Opt 17: 085003, 2012.

22. Vajaitu C, Draghici CC, Solomon I, Lisievici CV, Popa AV, Lupu M, Caruntu C, Constantin MM and Voiculescu VM: The central role of inflammation associated with checkpoint inhibitor treatments. J Immunol Res 2018: 4625472, 2018.

23. Yilmaz E and Borchert HH: Effect of lipid-containing, positively charged nanoemulsions on skin hydration, elasticity and erythema - an in vivo study. Int J Pharm 307: 232-238, 2006. 